\title{
Incidência de acidentes ofídicos por gêneros de serpentes nos biomas brasileiros
}

\author{
Incidence of venomous snakebite accidents by snake species in \\ Brazilian biomes
}

Rafael Rodrigues Matos (https://orcid.org/0000-0002-0926-6783) ${ }^{1}$

Eliane Ignotti (https://orcid.org/0000-0002-9743-1856) ${ }^{1}$

\footnotetext{
${ }^{1}$ Universidade do Estado de Mato Grosso. Av. Tancredo Neves 1095, Cavalhada III. 78217-900 Cárceres MT Brasil. matosrafael@outlook.com
}

\begin{abstract}
The supply of antidotes for the treatment of venomous snakebite accidents, as well as the training of the care team, should be structured according to the frequency and risks by geographical areas. The scope of this article is to analyze the trend of the incidence of snakebite accidents in Brazilian biomes between 2003 and 2012. It involved the ecological study by means of Prais-Winsten regression of the incidence of snakebites by the Bothrops, Crotalus, Lachesis and Micrurus species for the Brazilian biomes in the period from 2003 to 2012. The research revealed that the number of accidents increased from 26,082 in 2003 to 27,870 in 2012 with an increased demand for antidotes. The trend analysis of the incidence showed a stationary trend, except for the Crotalus species with an upward trend in the Brazilian aggregate. An upward trend was found in the Cerrado for the Crotalus, Lachesis and Micrurus species: in the Pampa for Crotalus; in the Caatinga for Lachesis and in the Atlantic Forest for Micrurus. The conclusion drawn is that the stationary trend observed for snakebite accidents in Brazil is due to the fact that the majority of these (87\%) are caused by snakes of the Bothrops species. However, it should be stressed that there is an increased risk of accidents with snakes of the Crotalus, Lachesis and Micrurus species.
\end{abstract}

Key words Snakebite, Epidemiology, Trend
Resumo O aporte de insumos para o tratamento de acidentes ofídicos, bem como o treinamento da equipe assistencial devem ser orientados de acordo com a frequência e riscos por áreas geográficas. O objetivo deste artigo é analisar a tendência da taxa de incidência de acidentes ofídicos segundo gênero de serpentes nos biomas brasileiros no período de 2003 a 2012. Estudo ecológico de tendência da taxa de incidência dos acidentes ofídicos por serpentes dos gêneros Bothrops, Crotalus, Lachesis e Micrurus para os biomas no período de 20032012, por meio de regressão de Prais-Winsten. $O$ número de acidentes passou de $26.082 \mathrm{em} 2003$ para 27.870 em 2012, com aumento de demanda por insumos. A análise de tendência da taxa de incidência mostrou tendência estacionária, exceto para o gênero Crotalus com tendência ascendente no agregado do Brasil. Tendência ascendente foi verificada no Cerrado para o gênero Crotalus, Lachesis e Micrurus, no Pampa também para Crotalus, na Caatinga para o gênero Lachesis e na Mata Atlântica para o gênero Micrurus. A tendência estacionária verificada para os acidentes ofídicos no Brasil decorre da maioria destes (87\%) serem causados por serpentes do gênero Bothrops. No entanto, destaca-se aumento do risco para acidentes com serpentes dos gêneros Crotalus, Lachesis e Micrurus.

Palavras-chave Picada de cobra, Epidemiologia, Tendência 


\section{Introdução}

Os acidentes com serpentes desde tempos remotos povoam o imaginário popular, sempre dotados de um significado místico ${ }^{1}$. Fato é que o homem e serpente sempre se relacionaram, e apesar de um não fazer parte da cadeia alimentar do outro, com raras exceções, os encontros casuais geralmente acabam com prejuízo para um dos lados, seja o homem ferindo ou matando a serpente, ou o contrário ${ }^{2}$. A Organização Mundial da Saúde (OMS) estimou 2,7 milhões de acidentes ofídicos com humanos anualmente no mundo ${ }^{3}$. No panorama global de incidência de ofidismo, o Brasil é o terceiro país, junto ao Vietnã, em número de acidentes com serpentes peçonhentas no mundo, atrás apenas de Îndia e Sri Lanka ${ }^{4}$. Por se tratar de um fenômeno que ocorre de forma muito mais vultosa em regiões tropicais, onde os indivíduos carecem de sistemas de saúde plenamente organizados e preparados, bem como meios para acessá-lo, este agravo foi incluído na Lista de Doenças Tropicais Negligenciadas ${ }^{3}$.

Quanto aos gêneros de serpentes peçonhentas que causam acidentes com humanos no Brasil incluem-se os da família Viperidae, destacandose a subfamília Crotalinae, à qual pertencem os gêneros Crotalus (cascavel), Bothrops (jararaca) e Lachesis (surucucu); e da família Elapidae, que engloba o gênero Micrurus, cujas espécies são conhecidas popularmente por corais verdadeiras ${ }^{5,6}$.

A diversidade de espécies que causam estes acidentes é determinada pela variedade de paisagens naturais no país, influenciada pelas características naturais intrínsecas e pelo clima. $\mathrm{O}$ espaço geográfico que guarda similitudes nestes aspectos é classificado como bioma, que neste estudo entende-se como uma região que detém uniformidade de ambiente classificado com base no macroclima, fitofisionomia, solo e altitude ${ }^{7,8}$.

Os acidentes ofídicos representam um grande problema de saúde pública para os países em desenvolvimento ${ }^{9}$, de forma que a taxa de incidência por 100 mil habitantes dos acidentes ofídicos no Brasil no ano de 2001 foi de 10,7, enquanto em 2013 foi de 14,3 $3^{10}$.

Apesar dos esforços significativos e bem-sucedidos no Brasil em acidentes ofídicos nas áreas de pesquisa, fabricação de antiveneno e controle de qualidade, treinamento de profissionais de saúde no diagnóstico e manejo clínico das picadas, pouco se sabe sobre os determinantes da incidência de acidentes ofídicos para planejar intervenções e reduzir o impacto desta condição médica. Entender a complexidade das interações ecológicas em uma região geográfica é importante para prever, prevenir e controlar medidas de acidentes ofídicos.

$\mathrm{O}$ aporte de insumos de soroterapia, bem como o treinamento da equipe assistencial deve ser dirigido pelas características próprias da região, prospectando a variação na taxa de incidência para os anos subsequentes, de acordo com a tendência, para que se possa oferecer tratamento em quantidade e qualidade adequadas. Este estudo tem por objetivo analisar a tendência da taxa de incidência de acidentes ofídicos segundo gênero de serpentes nos biomas brasileiros no período de 2003 a 2012.

\section{Método}

Estudo ecológico de análise de tendência da taxa de incidência dos acidentes ofídicos no Brasil segundo gêneros Bothrops, Crotalus, Lachesis e Micrurus para os biomas Amazônia, Caatinga, Cerrado, Mata Atlântica, Pampa e Pantanal, no período de 2003-2012.

Para o numerador da taxa de incidência utilizou-se o somatório das notificações de acidentes ofídicos de todos os municípios que compõem cada um dos biomas cuja fonte de dados foi o Sistema Nacional de Agravos de Notificação (SINAN).

Utilizou-se a classificação dos municípios brasileiros de acordo com os biomas, segundo o Instituto Brasileiro de Geografia e Estatística ${ }^{8}$. Para os municípios que apresentavam mais de um bioma em sua área territorial optou-se pelo bioma predominante na localização do centro urbano. Pelo fato de os biomas apresentarem mais homogeneidade fitofisionômica e climatológica em comparação com as divisões geográficas artificiais (estados e regiões), esta unidade espacial foi eleita como espaço de análise (Figura 1).

Os acidentes notificados sem especificação do gênero, que representaram em média 18,5\% do total de registros, foram distribuídos proporcionalmente, por método direto de estimativa, nos quatro gêneros descritos. As taxas por biomas foram calculadas por 100.000 habitantes, em função do total da população residente nos respectivos biomas e anos de análise, baseado em verificação e projeção do IBGE ${ }^{8}$.

Para a análise de tendência das taxas de incidência dos acidentes ofídicos utilizou-se o método de regressão de Prais-Winsten. O método foi selecionado por controlar a autocorrelação ano a ano. As Variações Percentuais Médias Anuais 
Figura 1. Biomas brasileiros.

Fonte: Ministério do Meio Ambiente, 2017

(APC) foram obtidas a partir do anti-log do estimador $\beta^{11}$.

$$
A P C=\left[-1+10^{\beta}\right] * 100 \%
$$

$O$ intervalo de confiança foi calculado por meio da equação:

IC $95 \%=\left[-1+10^{\beta \operatorname{Min}}\right] * 100 \% ;\left[-1+10^{\beta \mathrm{Max}}\right]^{*} 100 \%$

Os betas (Min e Max) são os limites inferiores e superiores dos IC95\% para cada beta estimado por meio do modelo de regressão.

O presente estudo recebeu parecer de dispensa do Comitê de Ética em pesquisa por se tratar de dados secundários, disponibilizados on-line pelo Ministério da Saúde.

\section{Resultados}

No período de 2003 a 2012, foram notificados 275.117 acidentes ofídicos envolvendo humanos no Brasil, com uma média anual de 27.511 acidentes. O principal gênero causador de acidentes foi o Bothrops (jararacas) com $86,8 \%$, seguido pelo gênero Crotalus (cascavel) com 8,9\% dos acidentes. O gênero Lachesis (surucucu) e Micrurus (coral) foram responsáveis respectivamente por 3,5 e $0,8 \%$ dos acidentes no período.
A Mata Atlântica e a Amazônia concentraram a maior parte dos acidentes ofídicos com humanos no período com $37 \%$ e $33 \%$, respectivamente, seguidas do bioma Cerrado com 18,9\% dos acidentes, Caatinga com 9,1\%, Pampa com 1,6\% e Pantanal com $0,6 \%$.

A análise pelo método de Prais-Winsten mostrou tendência estacionária para os acidentes ofídicos no Brasil no período de 2003 a 2012, quando a taxa de incidência por 100.000 habitantes foi 15,1 e 14,5. Em números absolutos, os valores notificados mínimos e máximos da série foram 25.532 acidentes em 2007 e 28.825 acidentes em 2011 (Gráfico 1).

\section{Acidentes botrópicos}

Apresentaram tendência estacionária para o Brasil tanto quanto para cada um dos biomas no período estudado (Gráfico 2).

No Brasil, os acidentes botrópicos mostraram um percentual de redução média anual de 0,4\% não significante. A taxa de incidência por 100.000 habitantes passou de 12,8 em 2003 (22.681 acidentes) para 12,3 em 2012 (23.320 acidentes).

Para a Amazônia verificou-se aumento da taxa de incidência por 100.000 habitantes de 


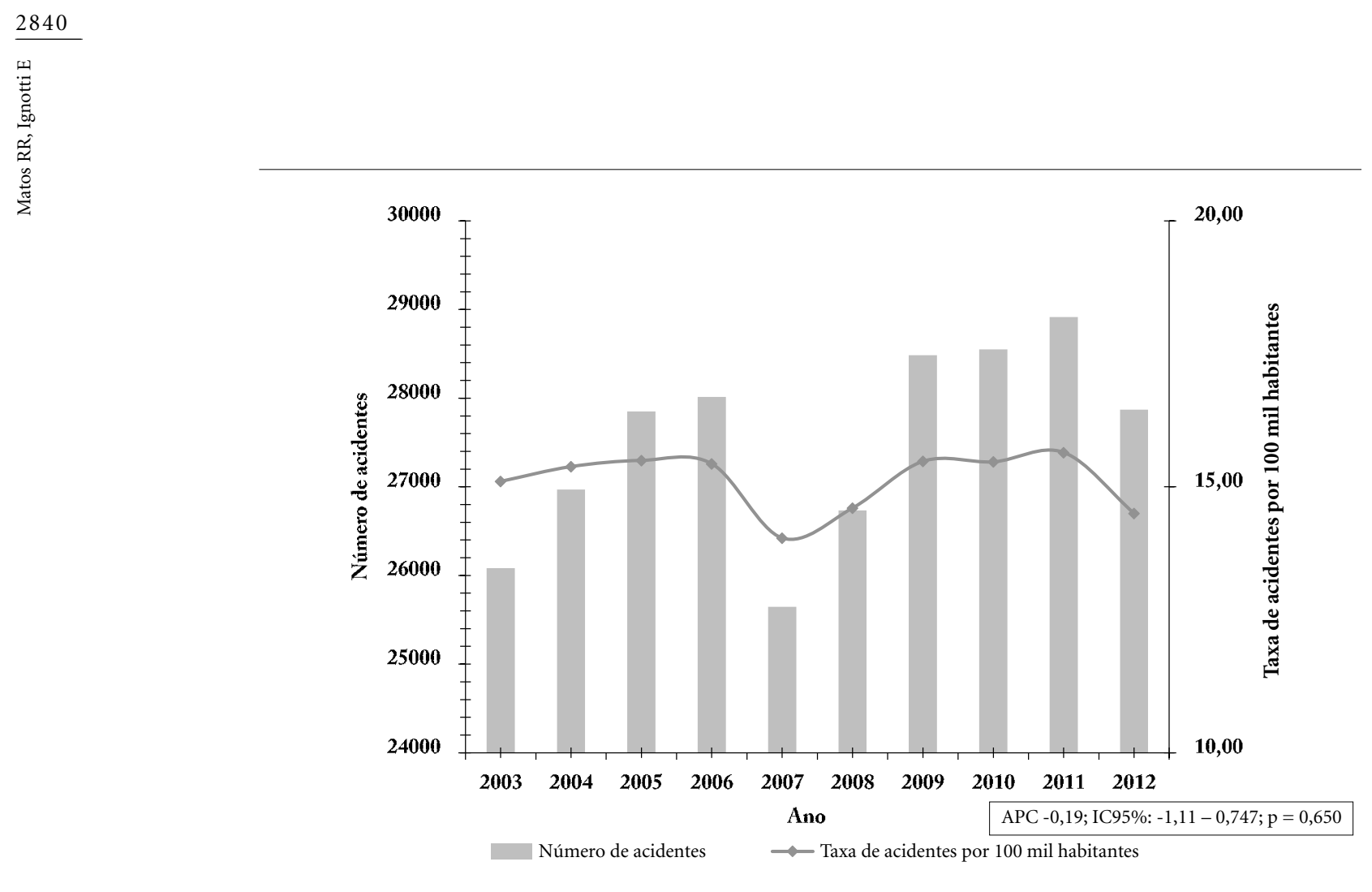

Gráfico 1. Variação percentual anual (APC) dos acidentes ofídicos no Brasil, 2003 a 2012.

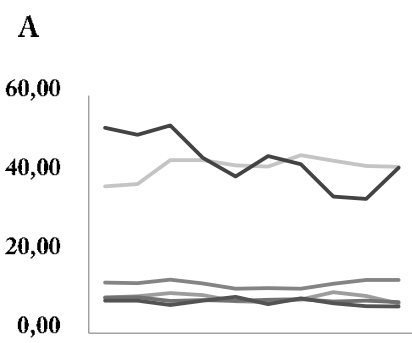

20032005200720092011

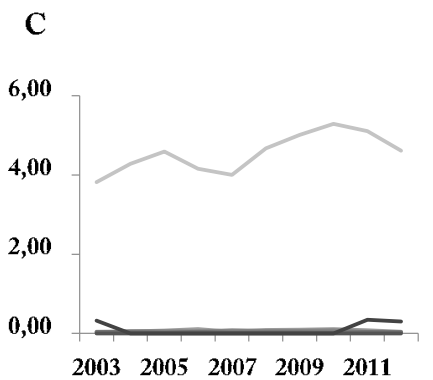

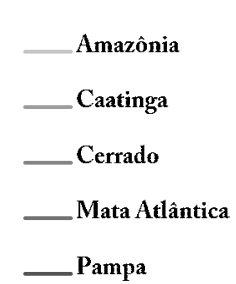

—Pampa

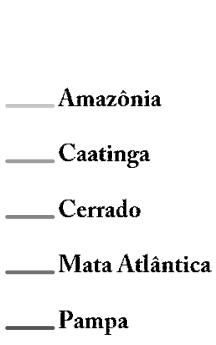

\section{B}

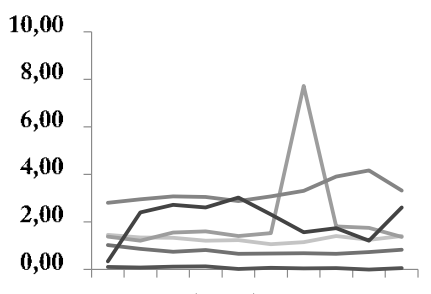

$20032005 \quad 2007 \quad 20092011$

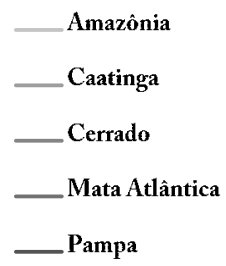

D

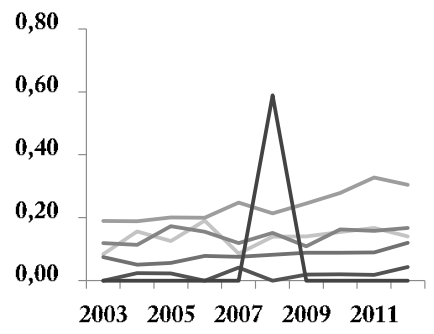

Amazônia

Caatinga

Cerrado

__ Mata Atlântica

_ Pampa

Gráfico 2. Evolução da taxa de incidência de acidentes ofídicos por gênero de serpentes e biomas brasileiros no período de 2003 a 2012. A) Gênero Bothrops; B) Gênero Crotalus; C) Gênero Lachesis; D) Gênero Micrurus. 
37 em 2003 (6.822 acidentes) para 42 em 2012 (8.405 acidentes), ou seja, cerca de quatro vezes a taxa média do país.

$\mathrm{Na}$ Caatinga a taxa de incidência por 100.000 habitantes partiu de 9 em 2003 (1.928 acidentes) alcançando 7,4 em 2012 (1.735 acidentes). Para o Cerrado a taxa de incidência apresentou pequena variação ao longo do período, passando de 12,7 (3.843 acidentes) para 13,4 (4.549 acidentes). No bioma Mata Atlântica, a taxa de incidência anual por 100.000 habitantes apresentou pequena redução de 8,9 (9.256 acidentes) para 7,8 (8.773 acidentes). Com 100.391 acidentes notificados o bioma Mata Atlântica apresentou o maior número de acidentes botrópicos.

No bioma Pampa os acidentes passaram de 8,3 por 100.000 habitantes em 2003 (442 acidentes) para 6,7 em 2012 (362 acidentes). No Pantanal observou-se a maior taxa de incidência dentre os biomas, com 51,8 acidentes por 100.000 habitantes em 2003 (174 acidentes) para 41,8 em 2012 (151 acidentes) e importante flutuação no período (Tabela 1 ).

\section{Acidentes crotálicos}

Verificou-se tendência crescente com o APC estatisticamente significante de 1\% (IC95\% 0,03 - 1,30). No período em estudo, verificou-se uma taxa de incidência por 100.000 habitantes de 1,3 em 2003 (2.434 acidentes) para 1,5 em 2012 (2.606 acidentes) (Gráfico 2).

A análise de tendência por biomas, resultou em aumento estatisticamente significante para o Cerrado e Pampa, que apresentaram incremento médio anual maior que 3\% ao ano. Para a Mata Atlântica e Amazônia, não foi observada tendência significante. A primeira com o maior número de acidentes crotálicos no período, quando foram registraos 8.314 acidentes com a taxa de incidência por 100.000 habitantes de 1 em 2003
(1.061 acidentes) e 0,82 em 2012 (922 acidentes) e a segunda com taxa de incidência de 1,4 em 2003 (247 acidentes) e 1,4 em 2012 (282 acidentes). O Cerrado com as maiores taxas de incidência por 100.000 habitantes de acidentes crotálicos em 2003 alcançou 2,8 (841 acidentes) e 3,3 em 2012 ( 1.122 acidentes). No Pampa, o bioma com a menor taxa de incidência do país apresentou 0,33 por 100 mil habitantes (Tabela 1 ).

\section{Acidentes laquéticos}

Com taxa de incidência por 100.000 habitantes de 0,4 em 2003 (726 acidentes) e 0,5 em 2012 (1.025 acidentes) apresentaram tendência estacionária (Gráfico 2).

Verificou-se tendência estatisticamente significante para os biomas Caatinga e Cerrado com tendência de incremento de 3\%. Na Caatinga, com 314 acidentes notificados no período, a incidência anual no decênio estudado foi de 0,06 acidentes por 100.000 habitantes. No Cerrado verificou-se taxa de incidência em 2003 de 0,02 (6 acidentes) e em 2012 de 0,03 (12 acidentes). A variação média anual para o período foi de $3,2 \%$.

A Amazônia, como reduto natural do gênero, concentrou $88,7 \%$ dos acidentes laquéticos do país e não foi verificada tendência. A taxa de incidência em 2003 foi de 3,82 e em 2012, 4,62 por 100.000 habitantes.

Na Mata Atlântica a taxa de incidência por 100.000 habitantes em 2003 foi de 0,03 (33 acidentes) e em 2012 foi de 0,02 (31 acidentes).Para o bioma Pampa não houve registro de acidentes com o gênero Lachesis no período em análise (Tabela 1).

\section{Acidentes micrúricos}

A taxa de incidência por 100.000 habitantes para os acidentes micrúricos no Brasil no perío-

Tabela 1. Variação Percentual Anual (APC) dos acidentes ofídicos por gêneros e biomas, Brasil (2003-2012).

\begin{tabular}{lrlrrrrrr}
\hline \multirow{2}{*}{ Local } & \multicolumn{2}{c}{ Bothrops } & \multicolumn{2}{c}{ Crotalus } & \multicolumn{2}{c}{ Lachesis } & \multicolumn{2}{c}{ Micrurus } \\
\cline { 2 - 9 } & APC & \multicolumn{1}{c}{ IC95\% } & APC & \multicolumn{1}{c}{ IC95\% } & APC & IC95\% & APC & \multicolumn{1}{c}{ IC95\% } \\
\hline Brasil & $-0,19$ & $-1,11 ; 0,74$ & 0,69 & 0,$03 ; 1,36$ & 3,28 & $-0,10 ; 6,77$ & 4,95 & $-0,03 ; 10,18$ \\
Amazônia & 1,15 & $-0,21 ; 2,54$ & $-0,46$ & $-0,99 ; 0,08$ & 2,8 & $-0,11 ; 5,79$ & 3,51 & $-0,15 ; 7,32$ \\
Caatinga & $-1,14$ & $-2,22 ; 0,06$ & 4,95 & $-0,1 ; 10,27$ & 3,04 & 0,$12 ; 6,04$ & 6,41 & $-0,46 ; 13,77$ \\
Cerrado & 0 & $-0,12 ; 0,12$ & 3,28 & 0,$06 ; 6,59$ & 3,28 & 0,$03 ; 6,63$ & 2,57 & 0,$00 ; 5,20$ \\
Mata Atlântica & $-1,14$ & $-2,5 ; 0,23$ & $-2,5$ & $-4,94 ; 0,00$ & $-1,83$ & $-3,74 ; 0,13$ & 6,91 & 0,$13 ; 14,14$ \\
Pampa & $-1,6$ & $-8,2 ; 4,58$ & 3,75 & 0,$07 ; 7,57$ & - & - & 21,3 & $-0,06 ; 38,09$ \\
Pantanal & $-4,06$ & $-8,24 ; 0,31$ & 5,93 & $-0,08 ; 12,29$ & $-4,72$ & $-9,22 ; 8,36$ & $-0,23$ & $-0,53 ; 0,07$ \\
\hline IC= Intervalo de Confiança 95\%;- = Não realizado. & & & & & &
\end{tabular}


do de 2003 a 2012 foi de 0,09 em 2003 (165 acidentes) e 0,14 em 2012 (280 acidentes). A análise de tendência não mostrou resultados estatisticamente significantes (Gráfico 2).

$\mathrm{Na}$ análise segundo biomas, verificou-se tendência estatisticamente significante para os biomas Cerrado e Mata Atlântica. No Cerrado o percentual de incremento médio anual foi de 2,6\%, onde a taxa de incidência de acidentes por 100.000 habitantes passou de 0,11 (36 acidentes) em 2003 para 0,17 (56 acidentes) em 2012. A Caatinga se destacou com as taxas mais elevadas de incidência, no entanto, com tendência estacionária.

Na Mata Atlântica houve tendência crescente média anual de $7 \%$ com taxa de incidência em 2003 de 0,07 e em 2012 de 0,11 por 100 mil habitantes, com 77 e 134 acidentes respectivamente.

A Tabela 1 apresenta os resultados das análises de tendência por meio de Variação Percentual Média Anual (APC) e respectivos intervalos de confiança em 95\% para os acidentes ofídicos com humanos por gênero das serpentes Bothrops, Crotalus, Lachesis e Micrurus e biomas brasileiros para o período de 2003 a 2012. Destaca-se que o bioma Cerrado apresentou tendência crescente para três gêneros, exceto Bothrops.

\section{Discussão}

Este é o primeiro estudo de análise de tendência da taxa de incidência dos acidentes ofídicos por gênero e biomas brasileiros. Para o país como um todo e o conjunto de gêneros de serpentes notificados foi observada tendência estacionária. No entanto, para a análise estratificada segundo gêneros e biomas, observou-se tendência ascendente particularmente no bioma Cerrado. No período de 10 anos estudado houve elevação da incidência dos acidentes ofídicos, que não resultou em aumento da taxa de incidência em razão do crescimento populacional. De todo modo, baseado na elevação de 26.082 acidentes em 2003 para 27.870 em 2012, trata-se de aumento na demanda por insumos para atendimento das vítimas.

Chippaux $^{10}$ analisou os acidentes com animais peçonhentos no Brasil por um período de 12 anos (2001-2012). Para os acidentes ofídicos descreveu uma taxa de incidência por 100.00 habitantes de 15,1 em 2003 e 14,3 em 2012, valores muito próximos dos obtidos neste estudo 15,1 e 14,5 respectivamente. $O$ autor não analisou a tendência para o período estudado, razão pela qual não foram realizadas comparações dos principais achados.
Os acidentes botrópicos apresentaram-se como os mais frequentes nos acidentes ofídicos para todos os biomas. Panorama coincidente com diversos estudos em outros espaços amostrais ${ }^{12-14}$. Esta observação pode ser explicada pelo fato de espécies de serpentes do gênero Bothrops ocuparem todos os principais ecossistemas, desde florestas tropicais até pradarias e outros habitats secos ${ }^{15}$.

Os acidentes com o gênero Crotalus foram os únicos a apresentar tendência de incremento no Brasil, bem como para os biomas Cerrado e Pampa e tendência de redução na Mata Atlântica. Os acidentes com o gênero Crotalus são os que apresentam maior mortalidade, sendo quatro vezes superior a mortalidade causada pelos acidentes com animais do gênero Bothrops $s^{6}$. Portanto, essa verificação de tendência de elevação destes acidentes pode representar aumento do risco de óbito em relação aos acidentes ofídicos em geral. Face ao fato de o gênero estar presente, sobremaneira, em regiões de campos abertos ${ }^{16}$, o aumento das taxas pode indicar uma relação com o processo de degradação da floresta, sendo substituída por pastagens ou campos agrícolas. Soma-se a isso a característica generalista do gênero Crotalus, quanto à ocupação de micro-habitat. Assim, espera-se que a espécie Crotalus durissus, única representante do gênero Crotalus no Brasil, seja encontrada em áreas antropizadas e provavelmente aproveite os efeitos do desmatamento ${ }^{17,18}$. Ruha et al. ${ }^{19}$ apontam um aumento dos casos registrados de acidentes com serpentes nos Estados Unidos da América no período de 2013 a 2015, incluindo o gênero Crotalus.

No Brasil, a mídia mantém constante divulgação de notícias acerca de captura de serpentes em áreas residenciais como noticiado por exemplo no município de Marília, área de Cerrado no interior de São Paulo ${ }^{20}$ e no Distrito Federal, região onde também predomina o Cerrado ${ }^{21}$. No primeiro caso, veicula-se que houve a captura de 107 serpentes em 68 dias do ano de 2018, dentre elas foram identificadas cascavéis e jararacas. Esta situação explicita um caso de coabitação por humanos e serpentes no mesmo ambiente, facilitando a interação fatídica entre ambos.

Os acidentes envolvendo o gênero Lachesis apresentaram tendência crescente para Caatinga e também no Cerrado. O maior número de acidentes ocorreu na Amazônia, fato este esperado em virtude de a Amazônia ser o habitat natural do gênero de acordo com Wen et $\mathrm{al}^{22}$, pois o gênero Lachesis ocorre em florestas ombrófilas densas $^{15}$. É provável que os registros de acidentes 
no Cerrado e Caatinga tenham ocorrido em municípios de fronteira com a Amazônia, local de atendimento e notificação destes casos. Ressalvase que o curso clínico dos acidentes com serpentes do gênero Lachesis, pode por vezes, ser confundido com aqueles dos acidentes por serpentes do gênero Botrops, o que tende a gerar confusão quanto à notificação.

Os acidentes micrúricos apresentaram tendência crescente para Cerrado e Mata Atlântica. De acordo com Bucaretchi et al. ${ }^{23}$ o gênero $\mathrm{Mi}$ crurus possui diversas espécies distribuídas por todo território brasileiro, em uma ampla gama de paisagens, o que sustenta a ocorrência de acidentes em toda extensão do país, ainda que em reduzido número quando comparados aos outros gêneros. Por estarem adaptadas à regiões antropizadas e selvagens, para serpentes deste gênero não se observa grandes variações na taxa de incidência dos acidentes micrúricos. Os acidentes com serpentes do gênero Micrurus, no Brasil, estão geralmente relacionados ao manejo desses animais, portanto, com menor influência de características sazonais ambientais ${ }^{24}$.

$\mathrm{Na}$ análise estratificada por biomas, verificou-se para o Cerrado tendência ascendente para os acidentes com os gêneros Crotalus, Lachesis e Micrurus. Dessa forma o Cerrado foi o bioma com maior aumento do risco de acidentes com serpentes.

Aragon et al. ${ }^{13}$ analisaram os acidentes ofídicos no estado de São Paulo, que apresenta grande extensão de Cerrado, no período de 2007 a 2014 e encontraram tendência de incremento para os acidentes com o gênero Bothrops e Crotalus, fato este, parcialmente observado pelo presente estudo.

O Cerrado é o segundo maior bioma brasileiro em extensão. Sua cobertura vegetal natural corresponde a $60,5 \%$ da área, sofrendo constantemente com a pressão agrícola e a antropização ${ }^{25}$. Estes fatores parecem ser os principais responsáveis pela elevação da taxa de incidência de acidentes ofídicos no bioma. Por outro lado, o bioma com maior número de acidentes por serpentes peçonhentas foi a Mata Atlântica, possivelmente devido a maior densidade populacional e pressão sobre o ambiente. Devido às particularidades fitofisionômicas e climatológicas de cada bioma, os gêneros com maior ou menor variação foram próprios de cada espaço, ainda que por vezes, com tendência estacionária para as taxas de incidência.

O Bioma Amazônia é constituído por um mosaico de fitofisionomias e características climatológicas. Observa-se ali uma floresta de terra firme, típico de floresta tropical pluvial, do Zo- nobioma I, que é predominante. Além deste, há a Floresta de Igapó, do Hidrobioma I, as Caatingas do Rio Negro e os Campos Rupestres ${ }^{26}$. Tais características contribuem para a observação de que foram notificados acidentes dos quatro gêneros. No que se refere ao gênero Lachesis, típico da região, Feitosa et al. ${ }^{27}$ quando analisaram apenas o estado Amazonas, encontraram uma taxa de incidência média de 11,5 casos de acidentes por grupo de 100.000 habitantes ao ano no período de 2007 a 2012. No presente estudo, para o bioma Amazônia, verificou-se a taxa de incidência por 100.000 habitantes variando de 3,8 em 2003 a 4,6 em 2012. É provável que restringido a análise a um ambiente central do bioma, como é o caso do estado Amazonas a taxa de incidência tende a aumentar, por se tratar de um gênero e seu próprio habitat. Para o gênero Bothrops, Feitosa et al. ${ }^{27}$ apontaram em média 35,5 casos por 100.000 habitantes ao ano entre 2007 e 2012 no estado Amazonas. Neste estudo, para o gênero Bothrops o bioma Amazônia apresentou taxa de incidência de 37 em 2003, e 42 em 2012. Quanto ao gênero Bothrops a taxa de incidência para o bioma é mais elevada do que a verificada para o estado do Amazonas. Tais diferenças decorrem possivelmente da inserção das áreas ecótones na análise.

Lima et al. ${ }^{28}$ mostraram que o gênero mais envolvido em acidentes com serpentes peçonhentas no estado do Amapá, no período de 2003 a 2006 foi o Bothrops, seguido pelos acidentes crotálicos e laquéticos, porém não analisaram a tendência destes acidentes. No presente estudo, observouse para o bioma Amazônia concentração dos acidentes no gênero Bothrops, seguido por Crotalus e Lachesis, e com menor magnitude o gênero Micrurus. No que tange ao gênero Crotalus, a distribuição espacial dos acidentes em toda a Amazônia brasileira mostra maior incidência em áreas de transição da floresta equatorial para a savana, e na própria savana, mas não necessariamente nas áreas intercaladas com florestas úmidas de folhas largas e florestas inundadas ${ }^{15}$. Este gênero tende a habitar áreas desmatadas, cujas características de sombra e cobertura vegetal se assemelham às do Cerrado ${ }^{29}$.

É fato que a estação chuvosa, a temperatura, o trabalho no campo/floresta e a expansão urbana para áreas periféricas contribuem para a ocorrência de acidentes ofídicos ${ }^{30}$. Neste contexto deve ser observado como possíveis fatores para justificar a elevada taxa de incidência de acidentes, sobretudo botrópicos, o avanço da fronteira agropecuária $^{31}$, desmatamento ${ }^{32}$ e a realização de grandes empreendimentos na floresta como 
a construção de hidrelétricas ${ }^{33}$. Situações que se fazem presentes no bioma há décadas, que modificam o habitat das serpentes e aumentam a exposição do indivíduo a estes animais.

A Caatinga é caracterizada como uma savana semiárida do Zonobioma II. Apresenta temperaturas elevadas e baixa e irregular pluviosidade, com intensa evapotranspiração ${ }^{26}$. Quando em 2017, Tavares et al..$^{34}$, analisaram os acidentes com serpentes no estado do Rio Grande do Norte, majoritariamente ocupado pelo bioma Caatinga, no período de 2007 a 2014 e observaram o gênero Bothrops como principal causador dos acidentes, em concordância com o presente estudo. No entanto não foi descrita a evolução da taxa de incidência anual para o estado.

A Caatinga, ainda, apesar de não abrigar animais do gênero Lachesis em seu domínio, por possuir áreas ecótones tanto com o bioma Amazônia, quanto com o bioma Mata Atlânti$\mathrm{Ca}^{26}$, apresentou acidentes com estes animais. De forma a ser este o único gênero para o qual se verificou tendência neste bioma. É provável que a ocupação das regiões de limite do bioma amazônico aumente o número de acidentes nessas áreas, sendo os acidentes atendidos em municípios onde predomina a Caatinga, elevando a notificação neste bioma.

A Mata Atlântica é formada por florestas do tipo Ombrófila Densa, Ombrófila Mista, Estacional Semidecidual, Estacional Decidual e Ombrófila Aberta, além de restingas. Está localizada ao extremo leste brasileiro, presente nos estados costeiros e avançando a partir daí para o interior do país $s^{35}$. Neste ambiente apresentaram tendência de redução dos acidentes com o gênero Crotalus e incremento dos acidentes micrúricos.

Chippaux ${ }^{14}$ aponta que a predominância dos acidentes crotálicos ocorre no Cerrado e leste do Brasil, região que abrange o bioma Mata Atlântica. No entanto, apesar de neste bioma estar concentrado a maior quantidade de acidentes com este gênero, houve no período redução da taxa de incidência. Mise et al. ${ }^{36}$ relatam alta incidência de acidentes ofídicos na Bahia, com predomínio de Mata Atlântica em seu litoral, e relaciona o evento com a produção de cacau e café, além da degradação do ambiente natural das serpentes. A Mata Atlântica é o bioma onde vive $70 \%$ da população brasileira, onde se concentra $80 \%$ do Produto Interno Bruto (PIB) e apresenta apenas $20 \%$ de áreas preservadas ${ }^{37}$, fatos que corroboram o achado de que está concentrado neste bioma a maior parte dos acidentes ofídicos no país.

O Pampa é um bioma restrito ao estado do Rio
Grande do Sul, e ocupa 63\% do território estadual. No entanto representa apenas $2 \%$ do território nacional. Apresenta como principal atividade econômica a pecuária extensiva sobre seus campos ${ }^{38}$. O Pampa foi o bioma brasileiro para o qual foi observado a maior variação da taxa de incidência para acidentes com animais do gênero Micrurus, ainda que não significante. Apesar de apresentar um grande incremento, o número em absoluto de acidentes foi pouco frequente. $\mathrm{O}$ gênero Crotalus reapresentou o maior incremento entre os biomas e tendência significante. Por se concentrar no extremo sul do país, com características de clima e relevo próprias, o Pampa não abriga indivíduos do gênero Lachesis, de forma que não foi registrado nenhum acidente com este grupo no período analisado. Os animais do gênero Bothrops, apesar de presentes no bioma não mostraram tendência estatisticamente significante. Não foram encontrados estudos que relacionem acidentes envolvendo serpentes peçonhentas e humanos neste bioma que possam ser comparados a este estudo.

O Pantanal é considerado uma das maiores áreas úmidas do planeta. É o menor bioma brasileiro, com seus domínios estendendo-se de Mato Grosso a Mato Grosso do Sul, ocupando 1,7\% do território nacional, com baixa densidade populacional e economia baseada na agropecuária ${ }^{39}$. Devido a atividade econômica predominantemente agropecuária/extrativista e turismo ocorrem taxas altas de incidência para os acidentes botrópicos, principal agente causador de acidentes ofídicos neste bioma. Sendo a média populacional do período 348.500 habitantes, a taxa de incidência sofre importante variação no período, ainda que seja um fenômeno de elevada magnitude. A relação inversa entre densidade populacional e taxa de incidência é compatível com o observado por Chippaux $^{10}$. O Pantanal, na última década tem sido ocupado por lavouras, sobretudo de soja, causando devastação de áreas nativas e intensificação do trabalho no campo nessas regiões ${ }^{40}$. Essa mudança da paisagem pode contribuir para a variação da taxa de incidência de acidentes com serpentes no bioma devido a degradação ambiental, mudando o perfil de animais que povoam a região e consequentemente o perfil epidemiológico do agravo com humanos. Mesmo sendo um dos biomas cuja biodiversidade é um importante atrativo para a atividade turística, não foram encontrados estudos que relacionem acidentes envolvendo serpentes peçonhentas e humanos no Pantanal.

Como limitações deste estudo destacam-se as próprias inerentes aos estudos com dados secundários, que dependem da qualidade dos registros 
como ação de vigilância em saúde. Assim, subnotificações podem mitigar a magnitude do evento. Essa limitação depende da qualidade e da organização dos serviços de saúde de cada localidade. Os registros relativos ao ano de 2007 parecem estar aquém do ocorrido, quando observado o comportamento da série. Tal fato poderia ter influenciado os resultados para alguns biomas e gêneros. Por essa razão, neste estudo foram testadas análises com a exclusão do ano de 2007 da série e não foi verificada tendência ascendente no conjunto dos dados para o país, o que parece confirmar os achados sobre a estabilização do risco de acidentes ofídicos no Brasil, particularmente para o gênero Bothrops. Ressalva-se a possível notificação de acidentes causados por colubrídeos como animais do gênero Bothrops, devido ao pródromo semelhante, o que pode magnificar os acidentes notificados deste gênero.

Os achados deste estudo apontam a relevância da manutenção serviços de saúde organizados para atender a demanda por cuidados de urgência, sobremaneira contando com uma logística voltada para o tipo de acidente mais incidente em cada área de abrangência segundo características ambientais.

Conclui-se que a tendência estacionária verificada para os acidentes ofídicos no Brasil decorre da maioria destes $(87 \%)$ serem causados por serpentes do gênero Bothrops. No entanto, destaca-se aumento do risco para acidentes com serpentes dos gêneros Crotalus, Lachesis e Micrurus, bem como, as particularidades segundo biomas.

\section{Colaboradores}

RR Matos: delineamento do trabalho, análise de dados, escrita do trabalho. E Ignotti: delineamento do trabalho, orientação metodológica, revisão do trabalho.

\section{Referências}

1. Ribeiro MG. Imaginário da serpente de A a Z. Campina Grande: EDUEPB; 2017.

2. Hartmann MT, Marques AO, Almeida-Santos SM. Reproductive biology of the southern Brazilian pitviper Bothrops neuwiedi pubescens (Serpentes, Viperidae). Amphibia-Reptilia 2004; 25(1):77-85.

3. World Health Organization (WHO). Snakebite under spotlight in Oxford [página na Internet]. 2017 [acessado 2017 Set 20]. Disponível em: http://www.who.int/ snakebites/news/Snakebite_under_spotlight_in_Oxford/en

4. Kasturiratne A, Wickremasinghe AR, Silva N, Gunawardena NK, Pathmeswaran A, Premaratna R, Savioli L, Lalloo DG, Silva HJ. The Global Burden of Snakebite: A Literature Analysis and Modelling Based on Regional Estimates of Envenoming and Deaths. PLoS Med 2008; 5(11):e218.

5. Lemos JC, Almeida TD, Fook SML, Paiva AA, Simões MOS. Epidemiologia dos acidentes ofídicos notificados pelo centro de assistência e informações toxicológicas de Campina Grande (Ceatox - CG), Paraíba. Rev Bras Epidemiol 2009; 12(1):50-59.

6. Fundação Nacional de Saúde (FUNASA). Ministério da Saúde (MS). Manual de diagnóstico e tratamento de acidentes por animais peçonhentos. $2^{\mathrm{a}}$ ed. Brasília: MS; 2001.

7. Walter H. Vegetação e Zonas Climáticas. São Paulo: E.P.U. Ltda; 1986.
8. Instituto Brasileiro de Geografia e Estatística (IBGE). Brasil em síntese [página na Internet]. [acessado 2017 Jun 10]. Disponível em: https://brasilemsintese.ibge. gov.br/territorio.html

9. Gutiérrez JM, Solano G, Pla D, Herrera M, Segura A, Vargas M, et al. Preclinical Evaluation of the Efficacy of Antivenoms for Snake bite Envenoming: State-ofthe-Art and Challenges Ahead. Toxins (Basel) 2017; 9(5):163.

10. Chippaux JP. Epidemiology of envenomations by terrestrial venomous animals in Brazil based on case reporting: from obvious facts to contingencies. J Venom Anim Toxins Incl Trop Dis 2015; 21:13.

11. Antunes JLF, Waldman EA. Trends and spatial distribution of deaths of children aged 12-60 months in São Paulo, Brazil, 1980-98. Bull World Health Organ 2002; 80(5):391-398.

12. Oliveira RR, Sousa ACR, Belmino JFB, Furtado SS, Leite RS. The epidemiology of envenomation via snakebite in the State of Piauí, Northeastern Brazil. Rev Soc Bras Med Trop 2015; 48(1):99-104.

13. Aragon DC, Queiroz JAM, Martinez EZ. Incidence of snakebites from 2007 to 2014 in the State of São Paulo, Southeast Brazil, using a Bayesian time series model. Rev Soc Bras Med Trop 2016; 49(4):515-519.

14. Chippaux JP. Incidence and mortality due to snakebite in the Americas. PLoS Negl Trop Dis 2017; 11(6):e0005662. 
15. Campbell JA, Lamar WW. The Venomous Reptiles of the Western Hemisphere. Comstock Publishing Associates/Cornell University Press, Ithaca e Londres; 2004.

16. Brasil. Ministério da Saúde (MS). Manual de Diagnóstico e Tratamento de Acidentes Ofídicos. Brasília: Centro de Documentação do Ministério da Saúde; 1988.

17. Sazima I, Haddad C. Répteis da Serra do Japi: Notas sobre a história natural. In: Morellato L. História $\mathrm{Na}$ tural Da Serra Do Japi: Ecologia E Preservação de Uma Área Florestal No Sudeste Do Brasil, Campinas. São Paulo: Unicamp e Fapesp; 1992. p. 212-326.

18. Bastos EGM, Araujo AF, Silva HR. Registros das cascavéis Crotalus durissus terrificus (Laurenti) (Serpentes, Viperidae) no Estado do Rio de Janeiro, Brasil: um possível caso de invasão facilitada pelo desmatamento Rev Bras Zool 2005; 22(3):812-815.

19. Ruha AM, Kleinschmidt KC, Greene S, Spyres MB, Brent J, Wax P, Padilla-Jones A, Campleman S, ToxIC Snakebite Study Group. The epidemiology, clinical course, and management of snakebites in the North American snakebite registry. J Med Toxicol 2017; 13(4):309-320.

20. Jornal da Cidade de Bauru (JCNET). Cobra coral, cascavel e jararaca: Marília já soma 107 capturas [página na Internet]. 2018 [acessado 2018 Jun 5]. Disponível em: https://www.jcnet.com.br/Regional/2018/03/cobra -coral-cascavel-e-jararaca-marilia-ja-passa-de-107capturas.html

21. Correio Brasiliense. Frequentadores encontram cobras no Parque Águas Claras [vídeo na Internet]. 2018 [acessado 2018 Jun 15]. Disponível em: http:// www.correiobraziliense.com.br/app/noticia/cidades/2018/02/20/interna_cidadesdf,661006/video-frequentador-encontra-cobra-no-parque-aguas-claras. shtml

22. Wen FH, Monteiro WM, Silva AMM, Tambourgi DV, Silva IM, Sampaio VS, Santos MC, Sachett J, Ferreira LCL, Kalil J, Lacerda M. Snakebites and scorpion stings in the brazilian amazon: identifying research priorities for a largely neglected problem. PLoS Negl Trop Dis 2015; 9(5):e0003701.

23. Bucaretchi F, Capitani EM, Vieira RJ, Rodrigues CK, Zannin M, Silva Jr NJ, Casais-e-Silva LL, Hyslop S. Coral snake bites (Micrurus spp.) in Brazil: a review. of literature reports. Clin Toxicol (Phila) 2016; 54(3):222-234.

24. Melgarejo AR. Serpentes Peçonhentas do Brasil. In. Cardoso JLC, França OSF, Wen FH, Málaque CMS, Haddad Jr V, organizadores. Animais peçonhentos no Brasil: Biologia, clínica e terapêutica dos acidentes. $2^{\mathrm{a}}$ ed. São Paulo: Sarvier; 2009. p. 42-70.

25. Sano EE, coordenador. Mapeamento de remanescentes de cobertura vegetal natural do Cerrado. Planaltina: EMBRAPA Cerrados; 2007.

26. Coutinho LM. O conceito de bioma. Acta Bot Bras 2006; 20(1):13-23

27. Feitosa EL, Sampaio VS, Salinas JS, Queiroz AM, Silva IM, Gomes AA, Sachett J, Siqueira AM, Ferreira LCL, Santos MC, Lacerda M, Monteiro W. Older Age and Time to Medical Assistance Are Associated with Severity and Mortality of Snakebites in the Brazilian Amazon: A Case-Control Study. PLoS One 2015; 10(7):e0132237.
28. Lima ACSF, Campos CEC, Ribeiro JR. Perfil epidemiológicos de acidentes ofídicos do estado do Amapá. Rev Soc Bras Med Tropic 2009; 42(3):329-335.

29. Tozetti AM, Martins M. Atividade diária e sazonal padrões de cascavel sul-americana ao ar livre (Crotalus durissus). An Acad Bras Cien 2013; 85(3):1047-1052.

30. Pinho FMO, Pereira ID. Ofidismo. Rev Assoc Med Bras 2001; 47(1):24-29.

31. Domingues MS, Bermann C. O arco de desflorestamento na Amazônia: da pecuária à soja. Amb Soc $S P$ 2012; XV(2):1-22.

32. Alencar A, Pereira C, Castro I, Cardoso A, Souza L, Costa R, Bentes AJ, Stella O, Azevedo A, Gomes J, Novaes R. Desmatamento nos Assentamentos da Amazônia: Histórico, Tendências e Oportunidades. Brasília: IPAM; 2016.

33. Bittencourt MT. A justiça ambiental e os grandes empreendimentos do setor elétrico na Amazônia paraense [dissertação]. Belém: Universidade Federal do Pará; 2015.

34. Tavares AV, Araújo KAM, Marques MRV, Vieira AA, Leite RS. The epidemiology of snakebite in the Rio Grande do Norte State, Northeastern Brazil. Rev Inst Med Trop 2017; 59:e52.

35. Brasil. Ministério do Meio Ambiente (MMA). Mata Atlântica [página na Internet]. [acessado 2016 Jul 26]. Disponível em: http://www.mma.gov.br/biomas/mata-atlantica

36. Mise YF, Lira-da-Silva RM, Carvalho FM. Agriculture and snakebite in Bahia, Brazil - An ecological study. Ann Agric Environ Med 2016; 23(3):416-419.

37. Brasil. Ministério do Meio Ambiente (MMA). Área Mata Atlântica é habitada por $70 \%$ da população brasileira [página na Internet]. 2013 [acessado 2017 Jun 15]. Disponível em: http://www.mma.gov.br/ informma/item $/ 9818-\%$ C3\%A1 rea-da-mata-atl\%C3\%A2ntica-\%C3\%A9-habitada-por-70-da-popula\%C3\%A7\%C3\%A3o-brasileira

38. Brasil. Ministério do Meio Ambiente (MMA). Pampa [página na Internet]. 2016 [acessado 2017 Jun 15]. Disponível em: http://www.mma.gov.br/biomas/ pampa

39. Brasil. Ministério do Meio Ambiente (MMA). Pantanal [página na Internet]. 2015 [acessado 2017 Jun 15]. Disponível em: http://www.mma.gov.br/biomas/ pantanal

40. Brasil. Senado Federal. Cultivo de soja ameaça o Pantanal, afirmam especialistas [página na Internet]. 2016 [acessado 2017 Jun 15]. Disponível em: https:// www12.senado.leg.br/noticias/materias/2016/09/21/ cultivo-de-soja-ameaca-o-pantanal-afirmam-especialistas

Artigo apresentado em 13/09/2018

Aprovado em 23/11/2018

Versão final apresentada em 25/11/2018 